Published in final edited form as:

Otol Neurotol. 2018 April ; 39(4): 515-517. doi:10.1097/MAO.0000000000001728.

\title{
PREVALENCE OF POTENTIAL HYBRID AND CONVENTIONAL COCHLEAR IMPLANT CANDIDATES BASED ON AUDIOMETRIC PROFILE
}

\author{
Adele M. Goman, Ph.D. \\ Department of Otolaryngology-Head \& Neck Surgery Johns Hopkins School of Medicine \\ Baltimore, Maryland \\ Center on Aging and Health Johns Hopkins Medical Institutions Baltimore, Maryland \\ agoman1@jhmi.edu
}

Camille C. Dunn, Ph.D. and Bruce J. Gantz, M.D.

Department of Otolaryngology-Head and Neck Surgery The University of lowa lowa City, lowa

Frank R. Lin, M.D., Ph.D.

Department of Otolaryngology-Head \& Neck Surgery Johns Hopkins School of Medicine

Baltimore, Maryland

Center on Aging and Health Johns Hopkins Medical Institutions Baltimore, Maryland

Department of Epidemiology Johns Hopkins University Baltimore, Maryland

Department Mental Health Johns Hopkins University Baltimore, Maryland

Department of Geriatric Medicine Johns Hopkins University Baltimore, Maryland

\begin{abstract}
To the Editor: Hearing loss affects over 28 million adults aged 60+ in the United States (1). Previous research $(1,2)$ has estimated the prevalence of hearing loss using pure-tone-average (PTA) thresholds. However, the prevalence of other audiometric profiles such as those that may be suitable for a hybrid cochlear implant (CI) have not been estimated. A hybrid CI is appropriate for individuals with mid-to-high frequency severe-to-profound hearing loss, yet normal-to-moderate hearing loss in the low-frequencies (3). This contrasts to conventional CI criteria which varies from moderate-to-severe to severe-to-profound PTA hearing loss (4). Knowing the prevalence of, and number of people with, these audiometric profiles is important. This study estimated the prevalence of an audiometric profile that would potentially meet criteria for a hybrid CI or a conventional CI among adults aged $60+$. The number of individuals expected to have these audiometric profiles in the near (2020), and distant future (2060), was also estimated.

Data from the National Health and Nutritional Examination Survey (NHANES) were analyzed. Participants were a representative sample of the non-institutionalized US
\end{abstract}

Conflicts of Interest: A.M.G. was previously a consultant to Cochlear Ltd. C.C.D. is on the Audiology Advisory Board for Med-EL and Earlens, is a consultant to Cochlear Ltd. and is Faculty at the Institute of Cochlear Implant Training. F.R.L. is a consultant to Cochlear Ltd., Boehringer-Ingelheim, and Amplifon. 
population. All participants aged 70+ in the 2005 to 2006 and 2009 to 2010 cycles, all participants aged 20 to 69 in the 2011 to 2012 cycle, and a random half-sample of participants aged 20 to 69 in the 2001 to 2002 and 2003 to 2004 cycles completed pure-tone air-conduction audiometry.

The analytic sample $(n=2,847)$ comprised all participants aged $60+$ who, for both ears, had a threshold recorded for each of $0.5,1,2,3$, and $4 \mathrm{kHz}$. The hybrid audiometric profile was defined as a threshold at $0.5 \mathrm{kHz}$ less than or equal to $60 \mathrm{~dB} \mathrm{HL}$, and a high-frequency $(2,3$, and $4 \mathrm{kHz}$ ) PTA threshold more than or equal to $75 \mathrm{~dB}$ HL in the better ear. This profile corresponds to current candidacy guidelines for the Cochlear Nucleus Hybrid system (Cochlear Ltd, Sydney, Australia) (3). We also explored the prevalence of a hybrid CI audiometric profile in the worse ear. A conventional $\mathrm{CI}$ audiometric profile was defined as a severe-to-profound hearing loss with a 4-frequency $(0.5,1,2$, and $4 \mathrm{kHz})$ PTA is more than $60 \mathrm{~dB}$ in the better ear (5).

Prevalence was estimated for each age decade. Population projections from the US census bureau (6) were used to estimate the number of individuals with these audiometric profiles in 2020 and 2060 assuming that prevalence estimates of hearing loss derived from NHANES 2001-2012 data remain unchanged in the future. Sample weights were used to account for the complex sampling design. Analyses were conducted in STATA version 12 (Stata Corp, College Station, TX).

Table 1 presents the prevalence and number of individuals with hybrid CI and conventional $\mathrm{CI}$ audiometric profiles by age decade. The prevalence of a better-ear hybrid $\mathrm{CI}$ audiometric profile is $0.7 \%$ among 60 to 69 year olds and increases to $4.5 \%$ in individuals' more than or equal to 80 years. The prevalence of a worse-ear hybrid CI audiometric profile is $2.9 \%$ among 60 to 69 year olds and increases to $8 \%$ in individuals' more than or equal to 80 years. For a better-ear conventional CI audiometric profile, a similar prevalence pattern is observed (1\% among 60-69 yr olds increasing to 6.8\% in individuals' $280 \mathrm{yr}$ ). Among those meeting better-ear audiometric criteria for either a hybrid or conventional CI, $30.6 \%$ met better-ear criteria for both devices. Thus, 2.38 million unique individuals aged $60+$ could benefit from an implantable hearing device in 2020 increasing to 4.64 million individuals aged $60+$ in 2060.

Our results demonstrate that an estimated $1.9 \%$ of adults aged 60 and older have a better-ear hybrid audiometric profile corresponding to 1.51 million in 2020 and 2.89 million in 2060. Furthermore, $4.9 \%$ of adults aged 60 and older have a hybrid audiometric profile in their worse ear and the number of individuals with this profile increases from 3.78 million in 2020 to 6.65 million in 2060 given the aging population. For a better-ear conventional CI audiometric profile, we estimate that $2.5 \%$ of adults aged 60 or older have such a profile, corresponding to 1.92 million individuals in 2020 and 3.79 million individuals in 2060. There was some overlap in individuals meeting criteria for both devices. Although an advantage of this analysis is the representative sample of US adults, our study has limitations. There may be some imprecision in estimates of the actual prevalence of individuals who would meet CI candidacy criteria because our estimates do not account for aided speech understanding which is another key criterion needed to determine candidacy 
for a hybrid or conventional CI (4). This study also assumes that the prevalence of hybrid and conventional $\mathrm{CI}$ audiometric profiles will not substantively change between now and 2060. This is a reasonable assumption given that hearing loss prevalence has changed minimally over the past 40 years $(7,8)$. In summary, the magnitude of our prevalence estimates highlights the large number of older adults who could potentially benefit from cochlear implantation and underscores the increased efforts that will be needed to effectively reach these individuals in the future.

\section{Acknowledgments}

Funding: This work is supported by the Eleanor Schwartz Charitable Foundation.

\section{REFERENCES}

1. Goman AM, Lin FR. Prevalence of hearing loss by severity in the United States. Am J Public Health 2016;106:1820-2. [PubMed: 27552261]

2. Lin FR, Niparko JK, Ferrucci L. Hearing loss prevalence in the United States. Arch Intern Med 2011;171:1851-2. [PubMed: 22083573]

3. Cochlear. Determining Candidacy: Nucleus Hybrid | Cochlear. Available at: http:// www.cochlear.com/wps/wcm/connect/us/for-j professionals/products/cochlear-nucleus-hybridimplant-system/candidacy. Accessed January 11, 2017.

4. Gifford RH. Who is a cochlear implant candidate? Hear J 2011;64: 16-22.

5. World Health Organization. Grades of Hearing Impairment. Available at: http://www.who.int/pbd/ deafness/hearing_impairment_-grades/en/. Published n.d. Accessed July 10, 2014.

6. United States Census Bureau. Population Projections: 2014 National Population Projections: Summary tables: Table 9. Available at: http://www.census.gov/population/projections/data/national/ 2014/summarytables.html. Published 2014. Accessed January 14, 2016.

7. Hoffman HJ, Dobie RA, Ko CW, Themann CL, Murphy WJ. Americans hear as well or better today compared with 40 years ago: hearing threshold levels in the unscreened adult population of the United States, 1959-1962 and 1999-2004. Ear Hear 2010; 31:725-34. [PubMed: 20683190]

8. NASEM (National Academies of Sciences, Engineering, and Medicine). Hearing Health Care: Priorities for Improving Access and Affordability. Washington, DC: The National Academies Press; 2016. doi: $10.17226 / 23446$. 
TABLE 1.

Prevalance of and number of individuals aged $60+$ with a hybrid ${ }^{a} \mathrm{CI}$ or conventional ${ }^{b} \mathrm{CI}$ audiometric profile

\begin{tabular}{lccccccc}
\hline & \multicolumn{2}{c}{ Hybrid CI Audiometric Profile (Better Ear) } & \multicolumn{2}{c}{ Hybrid CI Audiometric Profile (Worse Ear) } & Conventi \\
Age Decade & Prevalence (95\% CI) & N2020 (Millions) & N2060 (Millions) & Prevalence (95\% CI) & N 2020 (Millions) & N 2060 (Millions) & Prevalence (95\% CI) \\
\hline $60-69$ & $0.71(0.18,1.24)$ & 0.28 & 0.34 & $2.87(1.52,4.22)$ & 1.13 & 1.39 \\
$70-79$ & $2.57(1.60,3.54)$ & 0.64 & 1.03 & $6.35(4.53,8.17)$ & 1.59 & 2.54 & $2.47(1.27,3.68)$ \\
$80+$ & $4.46(2.82,6.10)$ & 0.59 & 1.52 & $8.00(6.17,9.84)$ & 1.06 & 2.72 & $6.80(4.70,8.90)$ \\
Total $60+{ }^{c}$ & $1.94(1.41,2.48)$ & 1.51 & 2.89 & $4.86(3.88,5.84)$ & 3.78 & 6.65 & $2.47(1.78,3.16)$ \\
\hline
\end{tabular}

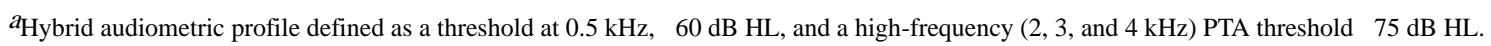

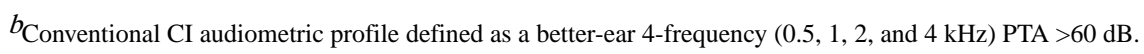

${ }^{\mathcal{C}}$ Total count is the sum of counts by age decade. Total count includes overlap in individuals meeting hybrid and conventional CI candidacy. In 2020, 1.05 million adults aged 60+ meet better-ear audiometric criteria for both devices, increasing to 2.04 million in 2060. The number of unique individuals meeting better ear audiometric criteria from implantable hearing devices is 2.38 million in 2020 and 4.64 million in 2060.

CI indicates cochlear implant; 95\% CI, 95\% confidence interval. 